\title{
CONTROLLABILITY OF A CLASS OF LINEAR SYSTEMS IN BANACH SPACE
}

\author{
QUAN ZHENG
}

(Communicated by Palle E. T. Jorgensen)

\begin{abstract}
This paper studies the notions of controllability for the linear systems associated with the generator of an exponentially bounded $C$-semigroup in a Banach space, controls also belonging to Banach spaces. Necessary and sufficient conditions are obtained in that framework, and the duality property is studied, which generalize the corresponding results of the linear systems associated with the generator of a strongly continuous semigroup.
\end{abstract}

\section{INTRODUCTION}

We will consider the linear control system

$$
x^{\prime}(t)=A x(t)+B u(t), \quad x(0)=x_{0}
$$

where $A$ is the generator of an exponentially bounded $C$-semigroup in a Banach space $X$ and $B$ is a bounded operator from a control Banach space $Y$ to $X$.

It is well known that (LCS) has received much attention, under the hypothesis that $A$ generates a strongly continuous semigroup (see, e.g., $[1,5,7,10]$ ). On the other hand, a generalization of strongly continuous semigroups, i.e., exponentially bounded $C$-semigroups, was recently introduced by Davies and Pang [3] and extended by Tanaka and Miyadera [9]; see also [2, 4, 6, 8]. In fact, there are many differential operators that generate $C$-semigroups but not strongly continuous semigroups.

This paper is organized as follows. Section 2 contains preliminary material on $C$-semigroups and inhomogeneous differential equations. Our main results are in $\S \S 3$ and 4. Finally, an example is given in $\S 5$ where strongly continuous semigroups cannot be applied but exponentially bounded $C$-semigroups can.

\section{Preliminaries}

Let $C$ be an injective operator in $B(X)$. A strongly continuous family $S(t)$ $(t \geq 0)$ in $B(X)$ is called an exponentially bounded $C$-semigroup (hereafter abbreviated as $C$-semigroup) on $X$, if $S(t+s) C=S(t) S(s)$ for $t, s \geq 0$, $S(0)=C$, and there exist constants $M, a$ such that $\|S(t)\| \leq M e^{a t}$ for $t \geq 0$.

Received by the editors January 21, 1992 and, in revised form, July 29, 1993.

1991 Mathematics Subject Classification. Primary 93C25, 34G10, 47D06.

Key words and phrases. Controllability, complete controllability, $C$-semigroup.

This project was supported by the National Science Foundation of China. 
It is known that $L_{r}:=\int_{0}^{\infty} e^{-r t} S(t) d t \quad(r>a)$ is injective in $B(X)$, and the closed linear operator $A$ defined by $A x=\left(r-L_{r}^{-1} C\right) x$ for $x \in D(A):=$ $\left\{x \in X ; C x \in R\left(L_{r}\right)\right\}$ is independent of $r$. We call it the generator of $S(t)$. Moreover, define

$$
\rho_{C}(A)=\{r ; R(C) \subset R(r-A) \text { and } r-A \text { is injective }\} .
$$

Lemma $2.1[4,9]$. Let $A$ generate a $C$-semigroup $S(t)$ satisfying $\|S(t)\| \leq$ $M e^{a t}$. Then

(a) $(a, \infty) \subset \rho_{C}(A)$. For every $r>a$ and $n \in N, R(C) \subset R\left((r-A)^{n}\right)$ and

$$
(r-A)^{-n} C=\frac{1}{(n-1) !} \int_{0}^{\infty} t^{n-1} e^{-r t} S(t) d t,
$$

which implies $\left\|(r-a)^{n}(r-A)^{-n} C\right\| \leq M$.

(b) $A=C^{-1} A C$, where $D\left(C^{-1} A C\right)=\{x \in X ; C x \in D(A)$ and $A C x \in$ $R(C)\}$.

(c) For every $x \in D(A)$ and $t \geq 0, S(t) A x=A S(t) x$ and $S(t) x=C x+$ $\int_{0}^{t} S(s) A x d s$.

The following proposition is a modification of Proposition 3.4 in [4], which will be useful to conclude that $A$ is the generator. The proof is easy and is omitted.

Proposition 2.2. A generates a $C$-semigroup $S(t)$ satisfying $\|S(t)\| \leq M e^{a t}$ iff $(a, \infty) \subset \rho_{C}(A), A=C^{-1} A C$, and (1) holds for $r>a$ and $n=1$. Moreover, in the "if" part, if we replace " $A=C^{-1} A C$ " with " $A \subset C^{-1} A C$ " then the generator of the $C$-semigroup $S(t)$ is $C^{-1} A C$.

Remark. A sufficient condition for " $A=C^{-1} A C$ " is " $\rho(A) \neq \varnothing$ and $A \subset$ $C^{-1} A C^{\prime \prime}$.

Now, let us turn to the inhomogeneous equation

$$
x^{\prime}(t)=A x(t)+f(t), \quad x(0)=x_{0}
$$

where $A$ generates a $C$-semigroup $S(t), f(\cdot) \in C([0, b], X)$. If $x(t)$ is a solution, i.e., $x(\cdot) \in C^{\prime}([0, b], X)$ and (2) is satisfied, then by Lemma $2.1(\mathrm{c})$

$$
\frac{d}{d s} S(t-s) x(s)=S(t-s) f(s) \text { for } 0 \leq s \leq t \leq b .
$$

Integrating this we obtain that $v(t) \in R(C)$ and $x(t)=C^{-1} v(t)$ for $0 \leq t \leq b$, where

$$
v(t)=S(t) x_{0}+\int_{0}^{t} S(t-s) f(s) d s \text { for } 0 \leq t \leq b .
$$

Theorem 2.3. Let $v(t)$ be defined by (3). Then the following statements are equivalent.

(a) $v(t) \in R(C)$ for $0 \leq t \leq b$ and $C^{-1} v(t)$ is a solution of (2).

(b) $v(t) \in R(C)$ for $0 \leq t \leq b$ and $C^{-1} v(\cdot) \in C^{\prime}([0, b], X)$.

(c) $v(t) \in C D(A)$ for $0 \leq t \leq b$ and $A C^{-1} v(\cdot) \in C([0, b], X)$.

Proof. Let $\|S(t)\| \leq M e^{a t}(t \geq 0)$ and $\alpha>a$. Define

$$
Y=\left\{x \in X ; C^{-1} S(\cdot) x \in C([0, \infty), X), \lim _{l \rightarrow \infty} e^{-\alpha t}\left\|C^{-1} S(t) x\right\|=0\right\},
$$




$$
\|x\|_{Y}=\sup _{t \geq 0} e^{-\alpha t}\left\|C^{-1} S(t) x\right\| \quad \text { for } x \in Y .
$$

Then (see [6]) $\left(Y,\|\cdot\|_{Y}\right)$ is a Banach space and $T(t) x:=C^{-1} S(t) x \quad(x \in Y)$ defines a strongly continuous semigroup on $Y$, with generator $A_{Y}$ (the part of $A$ in $Y)$. Moreover, $T(t) C x=S(t) x$ for all $x \in X$ and $C D(A) \subset D\left(A_{Y}\right)$.

(a) $\Rightarrow$ (b) This is trivial.

(b) $\Rightarrow$ (c) Let $u(t)=\frac{d}{d t} C^{-1} v(t)$ for $0 \leq t \leq b$. Then by (b)

$$
\begin{aligned}
& \left\|\frac{1}{h}(v(t+h)-v(t))-C u(t)\right\|_{Y} \\
& \quad \leq M\left\|\frac{1}{h}\left(C^{-1} v(t+h)-C^{-1} v(t)\right)-u(t)\right\| \rightarrow 0 \text { as } h \rightarrow 0,
\end{aligned}
$$

i.e., $v(\cdot) \in C^{\prime}([0, b], Y)$. It also follows from Theorem 2 in [12] that $v(t)$ is a solution of the equation

$$
y^{\prime}(t)=A_{Y} y(t)+C f(t), \quad y(0)=C x_{0} .
$$

Consequently

$$
C^{-1} v(t)=x_{0}+C^{-1} \int_{0}^{t} A_{Y} v(s) d s+\int_{0}^{t} f(s) d s .
$$

Hence by (b) $C^{-1} \int_{0}^{t} A v(s) d s \quad(t \geq 0)$ is differentiable. From the closedness of $C^{-1}$ we obtain $A v(t)=A_{Y} v(t) \in D\left(C^{-1}\right)=R(C)$ and $C^{-1} A v(t)=u(t)-$ $f(t) \in C([0, b], X)$. Therefore (c) follows from Lemma 2.1(b).

(c) $\Rightarrow$ (a) It is easy to see that $v(t)$ is a mild solution of (4). By (c) and Lemma 2.1(b), $v(t) \in C D(A) \subset D\left(A_{Y}\right)$ and $C^{-1} S(s) A_{Y} v(t)=S(s) A C^{-1} v(t)$ for $s \geq 0$ and $0 \leq t \leq b$. Thus

$$
\left\|A_{Y} v(t+h)-A_{Y} v(t)\right\|_{Y} \leq M\left\|A C^{-1} v(t+h)-A C^{-1} v(t)\right\| \rightarrow 0 \text { as } h \rightarrow 0 .
$$

It follows from Theorem 2 in [12] that $v(t)$ is a solution of (4). Consequently

$$
\begin{aligned}
v(t) & =C x_{0}+\int_{0}^{t} A_{Y} v(s) d s+C \int_{0}^{t} f(s) d s \\
& =C\left(x_{0}+\int_{0}^{t} A C^{-1} v(s) d s+\int_{0}^{t} f(s) d s\right),
\end{aligned}
$$

and therefore (a) follows.

From Theorem 2.3 one can deduce the following corollary (cf. [1, 12]).

Corollary 2.4. Let $x_{0} \in C D(A), f(t) \in R(C)$ for $0 \leq t \leq b$, and $C^{-1} f(\cdot) \in$ $L^{\prime}([0, b], X)$. Let one of the following conditions be satisfied:

(a) $C^{-1} f(t) \in D(A)$ for $0 \leq t \leq b$ and $A C^{-1} f(\cdot) \in L^{\prime}([0, b], X)$.

(b) There exists $g$ in $L^{\prime}([0, b], X)$ such that $C^{-1} f(t)=C^{-1} f(0)+\int_{0}^{t} g(s) d s$ for $0 \leq t \leq b$.

(c) $X$ is reflexive and, for every $t \in(0, b)$, there exist $M_{t}, l_{t} \geq 0$ such that

$$
\int_{0}^{t}\left\|C^{-1} f(s+h)-C^{-1} f(s)\right\| d s \leq M_{t} h \text { for } 0 \leq h \leq l_{t} .
$$

Then $v(t) \in R(C)$ for $0 \leq t \leq b$ and $C^{-1} v(t)$ is a solution of (2). 


\section{CONTROllability AND THE DUALITY PROPERTY}

This section concerns (LCS) with controls in $L^{p}([0, b], Y) \quad(1<p<\infty)$. Since a solution $x(t)$ of (LCS) satisfies $x(t) \in D(A)$ for $0 \leq t \leq b$, (LCS) in the general case cannot be steered to all of $X$. According to $\S 2$, we will choose to work with the following function

$$
v(t):=S(t) x_{0}+\int_{0}^{t} S(t-s) B u(s) d s \text { for } 0 \leq t \leq b
$$

where $S(t)$ is a $C$-semigroup generated by $A$.

We first introduce some notions of controllability of (LCS).

Definition 3.1. We say that (LCS) is

(a) exactly controllable on $[0, b]$ if for any $x_{0}, x_{1} \in X$, there exists a control $u \in L^{p}([0, b], Y)$ such that $v(b)=C x_{1}$.

(b) approximately controllable $[0, b]$ if, for any $x_{0}, x_{1} \in X$ and any $\varepsilon>0$, there exists a control $u \in L^{p}([0, b], Y)$ such that $\left\|v(b)-C x_{1}\right\|<\varepsilon$.

(c) exactly null controllable on $[0, b]$ if, for any $x_{0} \in X$, there exists a control $u \in L^{p}([0, b], Y)$ such that $v(b)=0$.

(d) approximately null controllable on $[0, b]$ if, for any $x_{0} \in X$ and any $\varepsilon>0$, there exists a control $u \in L^{p}([0, b], Y)$ such that $\|v(b)\|<\varepsilon$.

Since all subspaces of a finite-dimensional space are closed, the concepts introduced in Definition 3.1 are equivalent for finite-dimensional systems, but not in the general case. To derive necessary and sufficient conditions for these concepts, we need the following lemma (see [1]).

Lemma 3.2. Let $T_{i} \in B\left(X_{i}, X_{0}\right) \quad(i=1,2)$, where $X_{i}(i=0,1,2)$ are Banach spaces. Then:

(a) If $X_{i}(i=0,1,2)$ are reflexive then $R\left(T_{1}\right) \subset R\left(T_{2}\right)$ iff there exists $M>0$ such that $\left\|T_{1}^{*} x^{*}\right\|_{X_{1}^{*}} \leq M\left\|T_{2}^{*} x^{*}\right\|_{X_{2}^{*}}$ for all $x^{*} \in X_{0}^{*}$.

(b) $\overline{R\left(T_{1}\right)} \subset \overline{R\left(T_{2}\right)}$ iff $\operatorname{ker}\left(T_{2}^{*}\right) \subset \operatorname{ker}\left(T_{1}^{*}\right)$.

Theorem 3.3. (a) Let $X, Y$ be reflexive and $q=p /(p-1)$. Then $(L C S)$ is exactly controllable on $[0, b]$ iff there exists $M>0$ such that

$$
M \int_{0}^{b}\left\|B^{*} S(t)^{*} x^{*}\right\|_{Y^{*}}^{q} d t \geq\left\|S(b)^{*} x^{*}\right\|_{X^{*}}^{q}+\left\|C^{*} x^{*}\right\|_{X^{*}}^{q}, \quad \forall x^{*} \in X^{*} .
$$

(b) (LCS) is approximately controllable on $[0, b]$ iff $B^{*} S(t)^{*} x^{*}=0$ for $0 \leq t \leq b$ implies that $C^{*} x^{*}=S(b)^{*} x^{*}=0$.

(c) Let $X, Y$ be reflexive and $q=p /(p-1)$. Then (LCS) is exactly null controllable on $[0, b]$ iff there exists $M>0$ such that

$$
M \int_{0}^{b}\left\|B^{*} S(t)^{*} x^{*}\right\|_{Y^{*}}^{q} d t \geq\left\|S(b)^{*} x^{*}\right\|_{X^{*}}^{q}, \quad \forall x^{*} \in X^{*} .
$$

(d) (LCS) is approximately null controllable on $[0, b]$ iff $B^{*} S(t)^{*} x^{*}=0$ for $0 \leq t \leq b$ implies that $S(b)^{*} x^{*}=0$.

Proof. Define

$$
Q_{t} u=\int_{0}^{t} S(t-s) B u(s) d s \quad \text { for } u \in L^{p}([0, t], Y) .
$$


Then clearly $Q_{t} \in B\left(L^{p}([0, t], Y), X\right)$ and so $Q_{t}^{*} \in B\left(X^{*}, L^{q}\left([0, t], Y^{*}\right)\right)$. But

$$
\left\langle Q_{t} u, x^{*}\right\rangle=\int_{0}^{t}\left\langle u(s), B^{*} S(t-s)^{*} x^{*}\right\rangle d s, \quad \forall x^{*} \in X^{*}, u \in L^{p}([0, t], Y) .
$$

Therefore, $Q_{t}^{*}=B^{*} S(t-\cdot)^{*}$.

Now, from Definition 3.1(a) we know that (LCS) is exactly controllable on $[0, b]$ iff, for any $x_{0}, x_{1} \in X$, there exists $u \in L^{p}([0, b], Y)$ such that $C x_{1}=S(b) x_{0}+Q_{b} u$. Since $R\left(Q_{b}\right)$ is a subspace of $X$, this is equivalent to $R(C) \cup R(S(b)) \subset R\left(Q_{b}\right)$. Hence (a) follows from Lemma 3.2(a). Similarly the approximate controllability of (LCS) on $[0, b]$ is equivalent to $\overline{R(C)} \cup \overline{R(S(b))} \subset \overline{R\left(Q_{b}\right)}$ and so (b) follows from Lemma 3.2(b). Finally, from the proof of (a) and (b) one can deduce (c) and (d) respectively.

Remark. If $\overline{R(C)}=X$ or $\overline{R(S(b))}=X$, then (LCS) is approximately controllable on $[0, b]$ iff $B^{*} S(t)^{*} x^{*}=0$ for $0 \leq t \leq b$ implies $x^{*}=0$.

If $R(S(b)) \subset R(C)$, then (6) is equivalent to

$$
M \int_{0}^{b}\left\|B^{*} S(t)^{*} x^{*}\right\|_{Y^{*}}^{q} d t \geq\left\|C^{*} x^{*}\right\|_{X^{*}}^{q}, \quad \forall x^{*} \in X^{*} .
$$

Conversely, $R(C) \subset R(S(b))$ implies the equivalence of exact controllability and exact null controllability on $[0, b]$.

The following proposition gives a rank condition for approximate controllability.

Proposition 3.4. Let $\overline{\operatorname{span}}\left\{A^{n} B Y_{\infty} ; n=0,1,2, \ldots\right\}=X$, where $Y_{\infty}=\{u \in$ $\left.Y ; B y \in \bigcap_{n=1}^{\infty} D\left(A^{n}\right)\right\}$. Then (LCS) is approximately controllable on any $[0, b]$.

Proof. If $B^{*} S(t)^{*} x^{*}=0$ for $0 \leq t \leq b$, then, for every $y \in Y_{\infty},\left\langle S(t) B y, x^{*}\right\rangle=$ $0(0 \leq t \leq b)$. Differentiating this we see, using Lemma 2.1(c), $\left\langle S(t) A^{n} B y, x^{*}\right\rangle$ $=0(0 \leq t \leq b, n=0,1,2, \ldots)$ and therefore, by our assumptions, $S(t)^{*} x^{*}$ $=0$ for $0 \leq t \leq b$. The result now follows from Theorem 3.3(b).

We now turn to the duality property. To this end we need a result on the adjoint semigroup of a $C$-semigroup, which is a generalization of the Phillips's result (See [11].)

Theorem 3.5. Let $A$ generate a $C$-semigroup $S(t)$ on a reflexive Banach space $X$. If $\rho(A) \neq \varnothing$ and $\overline{R(C)}=X$, then $A^{*}$ generates the $C^{*}$-semigroup $S(t)^{*}$ on $X^{*}$.

Proof. We note first that the assumptions on $C$ imply that $C^{*}$ is an injective operator in $B\left(X^{*}\right)$ with dense range (see [11]) and that $D(A)$ is dense in $X$ (see [3]). By Lemma 2.1(b) we have $C A \subset A C$. It follows from the properties of adjoint operators (see [11]) that $C^{*} A^{*} \subset(A C)^{*} \subset(C A)^{*}=A^{*} C^{*}$, which implies $A^{*} \subset C^{*-1} A^{*} C^{*}$. Also $\rho(A) \neq \varnothing$ implies $\rho\left(A^{*}\right) \neq \varnothing$. Hence by the remark following Proposition 2.2 we obtain $A^{*}=C^{*-1} A^{*} C^{*}$.

Let $\|S(t)\| \leq M e^{a t}$ for $t \geq 0$. We next show $(a, \infty) \subset \rho_{C^{*}}\left(A^{*}\right)$ and $\left(r-A^{*}\right)^{-1} C^{*}=\left((r-A)^{-1} C\right)^{*}$ for $r>a$. In fact, let $L_{r}=(r-A)^{-1} C(r>a)$, then $C^{*}=\left((r-A) L_{r}\right)^{*} \supset L_{r}^{*}\left(r-A^{*}\right)$. On the other hand, $C^{*} \subset\left(L_{r}(r-A)\right)^{*}=$ $\left(r-A^{*}\right) L_{r}^{*}$. But $C^{*} \in B\left(X^{*}\right)$ and so $C^{*}=\left(r-A^{*}\right) L_{r}^{*}$. Therefore, the desired results follow. 
Finally, by (1) with $n=1$,

$$
\left\langle x,\left(r-A^{*}\right)^{-1} C^{*} x^{*}\right\rangle=\left\langle x,\left((r-A)^{-1} C\right)^{*} x^{*}\right\rangle=\int_{0}^{\infty} e^{-r t}\left\langle x, S(t)^{*} x^{*}\right\rangle d t
$$

for all $x \in X, x^{*} \in X^{*}$, and $r>a$. From Proposition 2.2 it remains to show the strong continuity of $S(t)^{*}$. Let $x^{*} \in D\left(A^{*}\right)$, then by Lemma 2.1(c)

$$
\left\langle x, S(t)^{*} x^{*}-C^{*} x^{*}\right\rangle=\int_{0}^{t}\left\langle x, S(s)^{*} A^{*} x^{*}\right\rangle d s, \quad \forall x \in D(A) .
$$

Since $S(s)^{*} A^{*} X^{*} \quad(s \geq 0)$ is weak *-continuous and since $X$ is reflexive, $S(\cdot)^{*} A^{*} x^{*} \in L_{\text {loc }}^{1}\left([0, \infty), X^{*}\right)$ and so

$$
S(t)^{*} x^{*}-C^{*} x^{*}=\int_{0}^{t} S(s)^{*} A^{*} x^{*} d s
$$

which implies that $S(\cdot)^{*} x^{*} \in C\left([0, \infty), X^{*}\right)$. The conclusion follows now from $\overline{D\left(A^{*}\right)}=X^{*}$ and $\left\|S(t)^{*}\right\|=\|S(t)\| \leq M e^{a t}(t \geq 0)$.

It is fairly well known that control and observation are "dual to each other". Dolecki and Russell [5] explore this duality relationship in an abstract Banach space setting. Following [5] (LCS) is also the abstract linear system

$$
Z_{2} \stackrel{F}{\rightarrow} Z_{1} \stackrel{E}{\leftarrow} D(E) \subset Z_{3}
$$

where $Z_{1}=X, Z_{2}=X \times X, Z_{3}=L^{p}([0, b], Y)$ and $E=Q_{b}, F(x, y)=$ $C y-S(b) x$. Let $X, Y$ be reflexive, $\rho(A) \neq \varnothing$ and $\overline{R(C)}=X$. Then it follows from [5] and Theorem 3.5 that the adjoint (observation) system of (LCS) is

$$
Z_{2}^{*} \stackrel{F^{*}}{\leftarrow} Z_{1}^{*} \supset D\left(E^{*}\right) \stackrel{E^{*}}{\rightarrow} Z_{3}^{*}
$$

where $Z_{1}^{*}=X^{*}, Z_{2}^{*}=X^{*} \times X^{*}, Z_{3}^{*}=L^{q}\left([0, b], Y^{*}\right)(q=p /(p-1))$ and $E^{*}=B^{*} S(b-\cdot)^{*}, F^{*}=\left(-S(b)^{*}, C^{*}\right)$, that is,

$$
y^{\prime}(t)=A^{*} y(t), \quad y(0)=y_{0}, \quad z(t)=B^{*} y(t) .
$$

Corresponding to (5) we may consider $C^{*} z(t)=B^{*} S(t)^{*} y_{0}$ for $0 \leq t \leq b$.

(LOS) is called continuously initially (resp. finally) observable on $[0, b]$ if (6) (resp. (7)) holds; (LOS) is called initially (resp. finally) observable on $[0, b]$ if $\bigcap_{0 \leq t \leq b} \operatorname{ker}\left(B^{*} S(t)^{*}\right)=\{0\} \quad\left(\right.$ resp. $\left.\bigcap_{0 \leq t \leq b} \operatorname{ker}\left(B^{*} S(t)^{*}\right) \subset \operatorname{ker}\left(S(b)^{*}\right)\right)$.

Now the duality theorem is a direct consequence of Theorem 3.3.

Theorem 3.6. (LOS) is continuously initially (resp. initially, continuously finally, finally) observable on $[0, b]$ iff $(L C S)$ is exactly (resp. approximately, exactly null, approximately null) controllable on $[0, b]$.

\section{Complete controllability}

This section concerns (LCS) with controls in $L_{\text {loc }}^{p}([0, \infty), Y) \quad(1<p<\infty)$. Definition 4.1. (LCS) is called completely controllable if, for any $x_{0}, x_{1} \in X$ and $\varepsilon>0$, there exists $t>0$ and a control $u \in L^{p}([0, t], Y)$ such that $\left\|v(t)-C x_{1}\right\|<\varepsilon$.

Theorem 4.2. The following statements are equivalent.

(a) (LCS) is completely controllable. 
(b) $\overline{\bigcup_{t>0} R(S(t))}=\overline{\bigcup_{t>0} R(S(t) B)}$.

(c) $B^{*} S(t)^{*} x^{*}=0$ for $t>0$ implies $S(t)^{*} x^{*}=0$ for $t>0$.

(d) $B^{*}\left((r-A)^{-1} C\right)^{*} x^{*}=0$ for $r>a$ implies $\left((r-A)^{-1} C\right)^{*} x^{*}=0$ for $r>a$.

If $X$ is a Hilbert space then (a) is also equivalent to

(e) $\int_{0}^{t} S(s) B B^{*} S(s)^{*} x d s=0$ for $t>0$ implies $S(t)^{*} x=0$ for $t>0$.

Proof. (a) $\Rightarrow$ (b) Since $v(t)=S(t) x_{0}+Q_{t} u(t>0)$, where $Q_{t}$ is defined by (8), the complete controllability of (LCS) is equivalent to $\overline{R(C)} \cup \overline{\bigcup_{t>0} R(S(t))} \subset$ $\overline{\bigcup_{t>0} R\left(Q_{t}\right)}$. But $\overline{\bigcup_{t>0} R\left(Q_{t}\right)} \subset \overline{\bigcup_{t>0} R(S(t) B)} \subset \overline{U_{t>0} R(S(t))}$ and, by the strong continuity of $S(t)$ on $t=0, \overline{R(C)} \subset \overline{U_{t>0} R(S(t))}$. Therefore, (b) follows.

(b) $\Rightarrow$ (c) If $B^{*} S(t)^{*} x^{*}=0$ for $t>0$, then $\left\langle x, x^{*}\right\rangle=0$ for all $x \in$ $\bigcup_{t>0} R(S(t) B)$. From (b) it follows that $\left\langle S(t) x, x^{*}\right\rangle=0$ for all $x \in X$ and $t>0$. Thus $S(t)^{*} x^{*}=0$ for $t>0$.

(c) $\Rightarrow$ (a) From the proof of (a) $\Rightarrow$ (b) it suffices to show that $\overline{\bigcup_{t>0} R(S(t))} \subset$ $\overline{\bigcup_{t>0} R\left(Q_{t}\right)}$. If this is not the case then for every $x_{0} \in \overline{\bigcup_{t>0} R(S(t))} \backslash \bigcup_{t>0} R\left(Q_{t}\right)$, there exists $x^{*} \in X^{*}$ such that $\left\langle x_{0}, x^{*}\right\rangle \neq 0$ and $\left\langle Q_{t} u, x^{*}\right\rangle=0$ for all $u \in$ $L^{p}([0, t], Y)$ and $t>0$. Since $Q_{t}^{*}=B^{*} S(t-\cdot)^{*}$ for $t>0$, it follows that $B^{*} S(t)^{*} x^{*}=0$ for $t>0$. By (c) we obtain $S(t)^{*} x^{*}=0$ for $t>0$. Hence, $\left\langle S(t) x, x^{*}\right\rangle=0$ for all $x \in X$ and $t>0$. This is a contradiction.

(c) $\Leftrightarrow$ (d) If $B^{*}\left((r-A)^{-1} C\right)^{*} x^{*}=0$ for $r>a$, then by (1) with $n=1$

$$
\int_{0}^{\infty} e^{-r t}\left\langle u, B^{*} S(t)^{*} x^{*}\right\rangle d t=\left\langle u, B^{*}\left((r-A)^{-1} C\right)^{*} x^{*}\right\rangle=0 \quad \forall u \in Y .
$$

It follows from the uniqueness of Laplace transform that $B^{*} S(t)^{*} x^{*}=0$ and so, by (c), $S(t)^{*} x^{*}=0$ for $t>0$. Again using (1) with $n=1$ we deduce $\left((r-A)^{-1} C\right)^{*} x^{*}=0$ for $r>a$. Similarly (d) implies (c).

(c) $\Leftrightarrow$ (e) If $\int_{0}^{t} S(s) B B^{*} S(s)^{*} x d s=0$ for $t>0$, then

$$
\int_{0}^{t}\left\|B^{*} S(s)^{*} x\right\| d s=\left(\int_{0}^{t} S(s) B B^{*} S(s)^{*} x d s, x\right)=0 \text { for } t>0,
$$

which implies $B^{*} S(t)^{*} x=0$ for $t>0$ and therefore, by (c), $S(t)^{*} x=0$ for $t>0$. It is clear that (e) implies (c).

Corollary 4.3. Let $\overline{R(C)}=X$. Then the following statements are equivalent.

(a) (LCS) is completely controllable.

(b) $\overline{U_{t>0} R(S(t) B)}=X$.

(c) $B^{*} S(t)^{*} x^{*}=0$ for $t>0$ implies $x^{*}=0$.

(d) $B^{*}\left((r-A)^{-1} C\right)^{*} x^{*}=0$ for $r>a$ implies $x^{*}=0$.

If $X$ is a Hilbert space then (a) is also equivalent to

(e) $\int_{0}^{t} S(s) B B^{*} S(s)^{*} x d s=0$ for $t>0$ implies $x=0$.

For most linear systems the operators $A$ and $B$ will be given rather than the $C$-semigroup $S(t)$. So it is important to obtain conditions for complete controllability involving the operators $A$ nd $B$. In this direction we give three conditions. The first is Theorem 4.2(d). The second is a rank condition (cf. Proposition 3.4), namely, $\overline{\operatorname{span}}\left\{A^{n} B Y_{\infty} ; n=0,1,2, \ldots\right\}=X$ implies the complete controllability of (LCS). Before we give the third condition we need the following result. 
Proposition 4.4. Let $A$ generate a $C$-semigroup $S(t)$ satisfying $\|S(t)\| \leq M e^{a t}$ for $t \geq 0$. Then for every $r_{0}>a,\left(r_{0}-A\right)^{-1}$ generates the $C$-semigroup

$$
T(t):=\sum_{n=0}^{\infty} \frac{t^{n}}{n !}\left(r_{0}-A\right)^{-n} C \text { for } t \geq 0
$$

satisfying $\|T(t)\| \leq M e^{t /\left(r_{0}-a\right)}$ for $t \geq 0$.

Proof. Let $A_{1}=\left(r_{0}-A\right)^{-1}$ and $a_{1}=\left(r_{0}-a\right)^{-1}$. We first show $C^{-1} A_{1} C=A_{1}$. Indeed, $A_{1} \subset C^{-1} A_{1} C$ follows easily from $A \subset C^{-1} A C$. Conversely, let $x \in D\left(C^{-1} A_{1} C\right)$ and put $y=C^{-1} A_{1} C$, then by Lemma 2.1(b) $x=r_{0} y-$ $C^{-1} A C y=\left(r_{0}-A\right) y$, i.e., $x \in D\left(A_{1}\right)$.

Next, let $r>a$; then $r_{0}-\frac{1}{r}>a$. Hence, it follows from $r-A_{1}=$ $r\left(r_{0}-\frac{1}{r}-A\right)\left(r_{0}-A\right)^{-1}$ that $r-A_{1}$ is injective and that $R\left(r-A_{1}\right)=$ $D\left(\frac{1}{r}\left(r_{0}-A\right)\left(r_{0}-\frac{1}{r}-A\right)^{-1}\right) \supset R(C)$. Therefore, $\left(a_{1}, \infty\right) \subset \rho_{C}\left(A_{1}\right)$.

Finally, by Lemma $2.1(\mathrm{a})$, we get $\|T(t)\| \leq M e^{a_{1} t}$ for $t \geq 0$ and

$$
\int_{0}^{\infty} e^{-r t} T(t) d t=\sum_{n=0}^{\infty} \int_{0}^{\infty} \frac{t^{n}}{n !} e^{-r t} d t A_{1}^{n} C=\sum_{n=0}^{\infty} r^{-n-1} A_{1}^{n} C=\left(r-A_{1}\right)^{-1} C
$$

for $r>a_{1}$. So the claim follows Proposition 2.2.

Theorem 4.5. Let $\|S(t)\| \leq M e^{a t}$ for $t \geq 0$. Then $(L C S)$ is completely controllable iff the linear system

$$
y^{\prime}(t)=\left(r_{0}-A\right)^{-1} y(t)+B u(t), \quad y(0)=y_{0}
$$

is completely controllable where $r_{0}>a$.

Proof. From Theorem 4.2 it suffices to show the equivalence of Theorem 4.2(c) and the following:

$\left(\mathrm{c}^{\prime}\right) \quad B^{*} T(t)^{*} x^{*}=0$ for $t>0$ implies $T(t)^{*} x^{*}=0$ for $t>0$.

We only show that $\left(\mathrm{c}^{\prime}\right)$ implies Theorem 4.2(c) (the converse implication is similar). Let $B^{*} S(t)^{*} x^{*}=0$ for $t>0$. Then it follows from (1) that

$$
\begin{aligned}
\left\langle u, B^{*} T(t)^{*} x^{*}\right\rangle & =\sum_{n=0}^{\infty} \frac{t^{n}}{n !}\left\langle\left(r_{0}-A\right)^{-n} C B u, x^{*}\right\rangle \\
& =\sum_{n=0}^{\infty} \frac{t^{n}}{n !} \int_{0}^{\infty} \frac{s^{n-1}}{(n-1) !} e^{-r_{0} s}\left\langle S(s) B u, x^{*}\right\rangle d s=0
\end{aligned}
$$

for all $u \in Y$. Namely, $B^{*} T(t)^{*} x^{*}=0$ for $t>0$ and so, by $\left(\mathrm{c}^{\prime}\right), T(t)^{*} x^{*}=0$ for $t>0$.

We now assume $r \in\left(a, r_{0}\right)$. Then

$$
\begin{aligned}
\int_{0}^{\infty} e^{-r t} S(t) d t & =(r-A)^{-1} C=\left(r_{0}-r\right)^{-1}\left(r_{0}-A\right)^{-1}\left(\left(r_{0}-r\right)^{-1}-\left(r_{0}-A\right)^{-1}\right)^{-1} C \\
& =-\left(r_{0}-r\right)^{-1} C+\left(r_{0}-r\right)^{-2}\left(\left(r_{0}-r\right)^{-1}-\left(r_{0}-A\right)^{-1}\right)^{-1} C \\
& =\left(r_{0}-r\right)^{-2} \int_{0}^{\infty} \exp \left\{-\left(r_{0}-r\right)^{-1} t\right\}(T(t)-C) d t
\end{aligned}
$$


here we note $\left(r_{0}-r\right)^{-1}>\left(r_{0}-a\right)^{-1}$. Since $T(t)^{*} x^{*}=0$ for $t>0$ implies $C^{*} x^{*}=0$, it follows that for every $x \in X$

$$
\begin{aligned}
\int_{0}^{\infty} & e^{-r t}\left\langle x, S(t)^{*} x^{*}\right\rangle d t \\
& =\left(r_{0}-r\right)^{-2} \int_{0}^{\infty} \exp \left\{-\left(r_{0}-r\right)^{-1} t\right\}\left\langle x, T(t)^{*} x^{*}-C^{*} x^{*}\right\rangle d t=0 .
\end{aligned}
$$

But $f(r):=\int_{0}^{\infty} e^{-r t}\left\langle x, S(t)^{*} x^{*}\right\rangle d t$ is analytic in $\{r ; \operatorname{Re} r>a\}$. Therefore, $f(r)=0(\operatorname{Re} r>a)$, and so the results desired follow.

Remark. As in $\S 3$, we call (LOS) completely observable if $\bigcap_{t>0} \operatorname{ker}\left(B^{*} S(t)^{*}\right)=$ $\{0\}$. Since $\overline{R(C)}=X$, the initial observability of (LOS) on $[0, b]$ implies the complete observability of (LOS). Moreover, (LOS) is completely observable iff (LCS) is completely controllable.

\section{AN EXAMPLE}

In this section we consider the following system of ordinary differential equations on $L^{2}(R) \times L^{2}(R)($ cf. $[3,8])$ :

$$
\left\{\begin{array}{l}
\frac{d}{d t} x_{1}(t, s)=i s^{p} x_{1}(t, s)+z s^{q} x_{2}(t, s)+b_{1}(s) u_{1}(t, s) \\
\frac{d}{d t} x_{2}(t, s)=i s^{p} x_{2}(t, s)+b_{2}(s) u_{2}(t, s) \quad \text { for } s \in R \text { and } t>0 \\
x_{1}(0, s)=x_{1}(s), \quad x_{2}(0, s)=x_{2}(s) \quad \text { for } s \in R
\end{array}\right.
$$

where $p, q$ are positive real numbers and $z$ is a nonzero complex number. Define

$$
A=\left(\begin{array}{cc}
i s^{p} & z s^{q} \\
0 & i s^{p}
\end{array}\right), \quad D(A)=\left\{(f, g) \in L^{2} \times L^{2} ; i s^{p} f+z s^{q} g, s^{p} g \in L^{2}\right\} .
$$

Then for $t>0$,

$$
e^{t A}=e^{i s^{p} t}\left(\begin{array}{cc}
I & z s^{q} t \\
0 & I
\end{array}\right), \quad D\left(e^{t A}\right)=\left\{(f, g) \in L^{2} \times L^{2} ; s^{q} g \in L^{2}\right\} .
$$

Therefore, $A$ is not the generator of a strongly continuous semigroup, but it is easy to see that $\{r ; \operatorname{Re} r \neq 0\} \subset \rho(A)$ if $q \leq 2 p$ and that $A$ generates a once $(q \leq p)$ or twice $(q \leq 2 p)$ integrated semigroup. In the general case $A$ generates a $C$-semigroup $S(t)$ where $C=\left(1+|s|^{q}\right)^{-1}\left(\begin{array}{ll}I & 0 \\ 0 & I\end{array}\right)$ and $S(t)=e^{t A} C$. Also, a simple computation yields

$$
C^{*}=C, \quad S(t)^{*}=e^{-i s^{p} t}\left(1+|s|^{q}\right)^{-1}\left(\begin{array}{cc}
I & 0 \\
\bar{z} s^{q} t & I
\end{array}\right) .
$$

Case 1. Let $b_{1}(s)=b_{2}(s)=1, u=\left(u_{1}, u_{2}\right) \in L^{2}\left([0, b], L^{2} \times L^{2}\right)$, and $B=$ $\left(\begin{array}{ll}I & 0 \\ 0 & I\end{array}\right)$. We show now that $(9)$ is exactly controllable on $[0, b]$. In fact, by Theorem 3.3(a), this is equivalent to

$$
\begin{aligned}
& M \int_{0}^{b} \int_{R}\left(1+|s|^{q}\right)^{-2}\left(|f(s)|^{2}+\left|\bar{z} s^{q} t f(s)+g(s)\right|^{2}\right) d s d t \\
& \quad \geq \int_{R}\left(1+|s|^{q}\right)^{-2}\left(2|f(s)|^{2}+|g(s)|^{2}+\left|\bar{z} s^{q} b f(s)+g(s)\right|^{2}\right) d s
\end{aligned}
$$


for all $f, g \in L^{2}$ and for some $M>0$. Calculating the terms in (10) yields

$$
\begin{aligned}
\int_{R}\left(1+|s|^{q}\right)^{-2}\left\{|z|^{2} s^{2 q} b^{2}(b M / 3-1)|f(s)|^{2}+(b M-2)\left(|f(s)|^{2}+|g(s)|^{2}\right)\right. \\
\left.+s^{q} b(b M / 2-1)(z \overline{f(s)} g(s)+\bar{z} f(s) \overline{g(s)})\right\} d s \geq 0 .
\end{aligned}
$$

But from $x^{2}+y^{2} \geq 2 x y$ we can deduce

$$
\left|s^{q} b(b M / 2-1)(z \overline{f(s)} g(s)+\bar{z} f(s) \overline{g(s)})\right| \leq|b M-2|\left(\frac{1}{4} b^{2} s^{2 q}|z f(s)|^{2}+|g(s)|^{2}\right) \text {. }
$$

Therefore, it is easy to check that if we choose $M=6 / b$ then (10) holds. So the claim follows.

Case 2. Let $b_{1}(s)=\delta \quad(=0$ or 1$), b_{2}(s)=1$, and $u_{1}=u_{2}=u \in L^{2}\left([0, b], L^{2}\right)$. Then $B \in B\left(L^{2}, L^{2} \times L^{2}\right)$ and $B u=(\delta u, u)$. From this, $B^{*} \in B\left(L^{2} \times L^{2}, L^{2}\right)$ and $B^{*}(f, g)=\delta f+g$. If

$$
B^{*} S(t)^{*}(f, g)=e^{i s^{p} t}\left(1+|s|^{q}\right)^{-1}\left(\delta f+\bar{z} s^{q} t f+g\right)=0 \text { for } 0 \leq t \leq b,
$$

then it follows that $f=g=0$. By Theorem 3.3(b) we know that (9) is approximately controllable on $[0, b]$. However, $(9)$ is not exactly null controllable on $[0, b]$. If this is not the case then by Theorem 3.3(c) there exists $M>0$ such that

$$
\begin{aligned}
& M \int_{0}^{b} \int_{R}\left(1+|s|^{q}\right)^{-2}\left|\delta f(s)+\bar{z} s^{q} t f(s)+g(s)\right|^{2} d s d t \\
& \quad \geq \int_{R}\left(1+|s|^{q}\right)^{-2}\left(|f(s)|^{2}+\left|\bar{z} s^{q} b f(s)+g(s)\right|^{2}\right) d s, \quad \forall f, g \in L^{2} .
\end{aligned}
$$

Choosing $g=-\delta f$ we obtain

$$
\int_{R}\left(1+|s|^{q}\right)^{-2}\left(\frac{1}{3} b^{3} M s^{2 q}|z|^{2}-1\right)|f(s)|^{2} d s \geq 0, \quad \forall f \in L^{2} .
$$

This is a contradiction if $\operatorname{supp} f \subset\left\{s \in R ;|s|<\left(b^{3} M|z|^{2} / 3\right)^{-1 / 2 q}\right\}$ and $f \neq 0$. Case 3. Let $b_{j}(s) \in L^{2}$ and $u_{j}(t, s)=u_{j}(t) \in L_{\text {loc }}^{2}(0, \infty) \quad(j=1,2)$. Then $B \in B\left(C \times C, L^{2} \times L^{2}\right)$ and $B\left(c_{1}, c_{2}\right)=\left(b_{1}(s) c_{1}, b_{2}(s) c_{2}\right)$. From this, $B^{*}(f, g)$ $=\left(\int_{R} f(s) \overline{b_{1}(s)} d s, \int_{R} g(s) \overline{b_{2}(s)} d s\right)$. Therefore, it follows from Theorem 4.2(c) that $(9)$ is completely controllable iff

$$
\left\{\begin{array}{l}
\int_{R} e^{-i s^{p} t}\left(1+|s|^{q}\right)^{-1} f(s) \overline{b_{1}(s)} d s=0, \\
\int_{R} e^{-i s^{p} t}\left(1+|s|^{q}\right)^{-1}\left(\bar{z} s^{q} t f(s)+g(s)\right) \overline{b_{2}(s)} d s=0
\end{array}\right.
$$

for $t \geq 0$ implies $f=g=0$. By Theorem 4.2(d) this is also equivalent to

$$
\left\{\begin{array}{l}
\int_{R}\left(r+i s^{p}\right)^{-1}\left(1+|s|^{q}\right)^{-1} f(s) \overline{b_{1}(s)} d s=0 \\
\int_{R}\left(r+i s^{p}\right)^{-1}\left(1+|s|^{q}\right)^{-1}\left(\bar{z} s^{q}\left(r+i s^{p}\right)^{-1} f(s)+g(s)\right) \overline{b_{2}(s)} d s=0
\end{array}\right.
$$

for $\operatorname{Re} r \neq 0$ implies $f=g=0$.

\section{REFERENCES}

1. R. F. Curtain and A. J. Pritchard, Infinite dimensional linear systems theory, Springer-Verlag, Berlin, 1978.

2. G. Da Prato, Semigruppi regolarizzabili, Ricerche Mat. 15 (1966), 223-248. 
3. E. B. Davies and M. M. H. Pang, The Cauchy problem and a generalization of the HilleYosida theorem, Proc. London Math. Soc. (3) 55 (1987), 181-208.

4. R. deLaubenfels, $C$-existence families and improperly posed problems, Semesterbericht Funktionalanlysis, vol. 17, Tübingen, Wintersemester, 1989/90, pp. 155-170.

5. S. Dolecki and D. L. Russell, A general theory of observation and control, SIAM J. Control Optim. 15 (1977), 185-220.

6. I. Miyadera and N. Tanaka, A remark on exponentially bounded $C$-semigroups, Proc. Japan Acad. Ser. A 66 (1990), 31-34.

7. D. L. Russell, Controllability and stabilizability theory for linear partial differential equations: Recent progress and open questions, SIAM Review 20 (1978), 639-739.

8. N. Tanaka, On perturbation theory for exponentially bounded C-semigroups, Semigroup Forum 41 (1990), 215-236.

9. N. Tanaka and I. Miyadera, Exponentially bounded C-semigroups and integrated semigroups, Tokyo J. Math. 12 (1989), 99-115.

10. K. N. Wang, Controllability and observability for distributed parameter control systems, Acta Math. Sci. 2 (1982), 403-420. (Chinese)

11. K. Yosida, Functional analysis, Springer-Verlag, Berlin, 1966.

12. Q. Zheng, Global solutions of abstract differential equations and applications, Acta Math. Sci. 11 (1991), 225-233. (Chinese)

Department of Mathematics, Huazhong University of Science and Technology, Wuhan 430074, People's Republic of China 